\title{
A bipartite patella caused anterior knee pain in an older adult: a case report
}

\section{Introduction}

Patella placed between the quadriceps and patellar tendons is the largest sesamoid bone in human body. It usually develops from a single ossification center. It is hypothesized that if this fusion does not occur, two or more ossification centers remain. In some cases It develops two (bipartite) or more ossification centers. ${ }^{1}$ Bipartite patella (BP) is generally an asymptomatic condition and is an incidental finding in knee radiographs. It reported incidance between $0,2-6 \%$ and, causes rarely anterior knee pain. Because patella plays an important role in knee biomechanics. Population based studies suggest that people having bipartite patella seen in male mostly are unaware of this condition. In the other hand There is localized patellar tenderness and mild swelling seen in young athletic people. It may be the only anatomical abnormality. In cases of patellar fracture there may be hemarthrosis, and restricted knee motion. X-ray of the patella is diagnostic and shows a linear regular radiolucent line. ${ }^{2} \mathrm{BP}$ has 3 classification criterias, Type $1(5 \%)$ is characterised by a transverse split and the accessory centre at the inferior pole, in type $2(20 \%)$ there is a longitudinal split and the accessory centre is found at the lateral margin of the patella, and in type $3(75 \%)$ the accessory centre is found at the superolateral pole of the patella. ${ }^{3}$ Treatment strategies including usually 3-4 weeks of rest from activity and also conservative treatment physical modalities and surgial interventions. ${ }^{2}$ In the lights of literature, painful bipartite patella is unknown since only case reports or case series. We aimed to present an older adult with bipartite patella without any sports.
Volume II Issue I - 2019

\author{
Yalkın Çalık \\ Bolu PM\&R Hospital,Turkey
}

Correspondence: Yalkın Çalık, Bolu PM\&R Hospital,Turkey, Emailyclk04@gmail.com

Received: October 14, 2017| Published: February 18, 2019

\section{Case report}

A 45 year-old male patient has suffered from anterior knee pain in unilateral side of right knee. Pain has been becomed for a year without any traumatic events. In physical examination; patellar tenderness was positive and palpation of patella was sensitive. VAS scores 2 in rest and 5 in motion. Range of movements of knee were normal. Laboratory findings including blood count, acute phase reactans (CRP,ESR) were normal. Antero-lateral right knee radiograms showed that he has bipartite patella with superolateral location (Figure 1). We planned other imagining methods (joint MRI of knee) for controlling diagnosis. After all, patient was taken in conservative treatment protocols including rest, anti-inflammatory medications (NSAID's) and physiotherapy modalities during 3 weeks. After treatment protocols he felt himself very well. Both VAS scores decreased. Palpation was normal. Patient has been followed up for pain and examination.

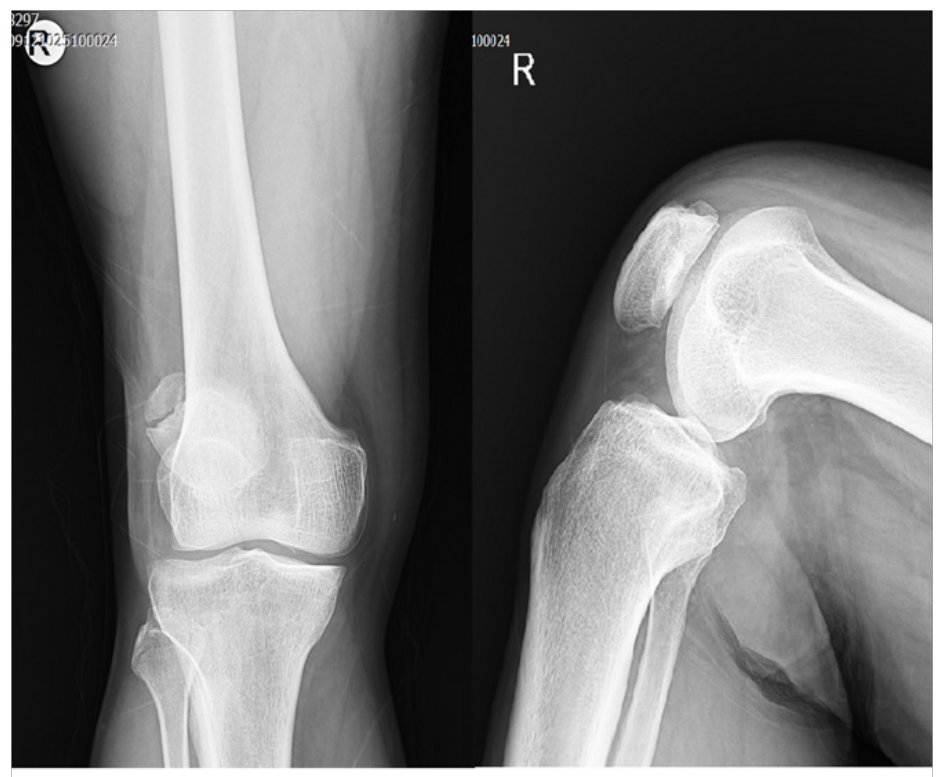

Figure I X-Ray of superolateral location with bipartite patella.

\section{Discussion}

For most anterior knee pain syndromes, the exact cause of pain is still unknown. In recent studies claimed that one of them is BP. ${ }^{1}$ Its incidence is reported as 1 to $2 \%$ in the population. Only $2 \%$ of patients with BP have symptoms such as anterior knee pain, tenderness on palpation of the patella. ${ }^{2}$ The proposed source of pain is the gap between bony fragments. In similar studies have described to be 
caused by fracture through or seperation of, synchondrosis between the accessory fragment and patella. ${ }^{1}$ The large majority of patients are successfully managed with conservative treatments, such as rest, non-steroidal anti-inflammatory medication, immobilisation and physiotherapy. When these methods are fail, surgical management include arthroscopic excision of the painful fragment, lateral retinacular release, vastus lateralis release, and internal fixation with or without bone grafting. ${ }^{2}$ Open reduction and internal fixation of the accessory fragment is invasive and may be considered overtreatment. ${ }^{4}$ In some studies as similar as ours, patients with BP were immobilized for 3 weeks, then patients returned to full sporting activity within 3 months. ${ }^{5}$ In another study dedicated that rest and anti-inflammatory medication were used for 2 weeks. After 2 years follow-up, patients with BP were asymptomatic. ${ }^{6}$ Nine patients who had a release of a type 3 bipartite patella were all able to resume activities within 2 months and reported 'excellent' results at final follow-up (2-9 years) with osseous union seen in eight of nine. The three patients who had a release of a type 2 fragment had a slower recovery. Activities were resumed in 6 months, two patients had an 'excellent' result, and one had a 'good' result at final follow-up (3-4 years). ${ }^{3}$ In a study of five patients, all patients had superolateral location of BP as ours and some of these patients treated with conservative strategies at least 6 months. Others had surgical events (open reduction, internal rotation). ${ }^{4}$ Our patient was older than other cases reported in the studies. They were almost young athlets but ours wasn't. We wanted to present an older adult with $\mathrm{BP}$ in the reviewed literature.

\section{Conclusion}

Although BP is seen rarely, Clinicians should examinethe patients carefully. Conservative treatment is choosed at first approach.

\section{Acknowledgements}

None.

\section{Conflicts of interest}

None.

\section{References}

1. Zabierek S, Zabierek J, Kwapisz A, et al. Bipartite patella in 35 year-old fitness instructor:a case report. Int J Sports Phys Ther. 2016;11(5):777783.

2. Patel DR, Villabolos A. Evaluation and management of knee pain in young athletes:overuse injuries of the knee. Transl Pediatr. 2017;6(3):190-198.

3. Mcmahon SC, LeRoux JA, Smith TO, et al. The management of painful bipartite patella: A systemic review. Knee Surg Sports Traumatol Arthrosc. 2016;24(9):2798-7805.

4. Vaishya R, Chopra S, Vijay V, et al. Bipartite patella causing knee pain in young adults: a report of 5 cases. J Orthop Surg (Hong Kong). 2015;23(1):127-130.

5. Okuno H, Sugita T, Kawamata T, et al. Traumatic separation of a type I bipartite patella: a report of four knees. Clin Orthop Relat Res. 2004;420:257-260.

6. Marya KM, Yadav V, Devagan A, et al. Painful bilateral bipartite patellae-case report. Indian J Med Sci. 2003;57(2):66-67. 